\title{
Quality of Service (QoS) and Quality of Experience (QoE) of the 4G LTE Perspective
}

\author{
Settapong Malisuwan, Dithdanai Milindavanij, and Wassana Kaewphanuekrungsi
}

\begin{abstract}
The increasing uptake of Internet of Things (IoT), Big data and cloud-based services introduces a new set of requirements for network performance. Furthermore, the evolution of mobile networks towards an all-IP 4G LTE introduces new challenges for traditional voice and data services. It is critical for operators to guarantee minimum levels of performance. Therefore, operators need to understand and manage both quality and performance of the services to fulfill on the technical quality of service $(\mathrm{QoS})$ as well as on the quality of experience (QoE) level. 4G LTE broadband mobile technologies have been designed with different QoS (Quality of Service) frameworks to enable delivery of the evolving Internet applications. Specifically, it is fundamental requirement to provide satisfactory service delivery to users and also to manage network resources. To provide QoS, different service levels are specified for different types or stream of traffic in term of throughput, latency (delay), jitter (delay variation) and packet errors or loss. This paper aims to provide a basic principle of QoS of the 4G LTE service. The impact of QoS parameter on QoE such as real-time and video communication services is discussed in this paper. QoS for 5G and M2M networks is also discussed in this paper as a future research.
\end{abstract}

Index Terms-Quality of service $(\mathrm{QoS})$, quality of experience (QoE), 4G, LTE, mobile, subscriber.

\section{INTRODUCTION}

Long Term Evolution (LTE) refers to the standard, developed by the Third Generation Partnership Project (3GPP), with the aim of creating new technology that enables high-speed data transmission and reception in mobile communication networks. It is currently considered a concrete 4G LTE technology after $2 \mathrm{G}$ and $3 \mathrm{G}$. By designing the technology deliberately, service providers are able to improve the existing systems to smoothly support LTE, no matter what networks they have originally belonged to: 3GPP (GSM, WCDMA, HSPA, etc.) or others (CDMA, WiMax, etc.) [1]. The development of the 4G LTE technology shows in Fig. 1.

In the mobile internet era, what consumers demand will vary based on the services offered and so will how they expect to experience the services. $4 \mathrm{G}$ promises to offer a vast range and diversity of converged devices, services and networks to revolutionize the way we communicate.

The LTE technology is designed to be capable of transmitting data at high speeds, giving low-latency response, and supporting large amounts of data simultaneously, even

Manuscript received February 12, 2016; revised April 27, 2016.

The authors are with the National Broadcasting and Telecommunications Commission (NBTC), Thailand (e-mail: settapong.m@nbtc.go.th, dithdanai.m@nbtc.go.th,puiwassana@gmail.com) with limited frequency resources (high spectral efficiency is achieved). These will promote the emergence of several new services or businesses.

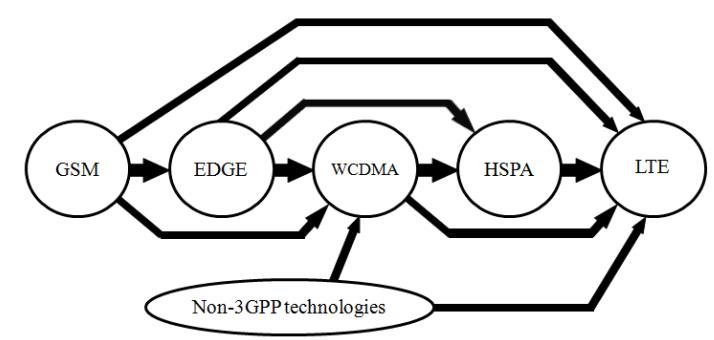

Fig. 1. Trends of network development toward LTE.

Other than utilizing the new type of radio air interface, network providers also have to convert their existing networks, both packet switching and circuit switching, into all-IP networks so as to achieve extremely high spectral efficiency. Although this requires heavier investments, it enables provision of diversified services arising from a perfect combination of voice, video, data transmission and reception. Besides, it can also be efficiently used with other fixed and wireless networks.

Due to its several special characteristics, LTE has gained extremely widespread acceptance worldwide. Nowadays, a lot of customers have become acquainted with using high-speed broadband networks at home and in office. In addition, the rapid growth in smartphones, tablet computers, netbooks, air cards, and various mobile phone applications also take part in enhancing the needs and expectations for broadband services in wireless networks as a result [2]. With the aid of LTE, consumers will be able to efficiently access various services though high-speed Internet, no matter where they are.

Another factor promoting the success in LTE is the way that network providers are able to gradually build up all-IP networks for LTE. In this regard, such all-IP networks are able to be used concurrently with the existing circuit-switched networks and enable the service providers to provide their services through LTE without the need for extremely heavy investments [3].

Technically, the key objective of LTE development is to increase speeds of data transmission and reception, and lower the response latency of the system so as to support "real-time" applications: game, VoIP, video conferencing, etc.

Regarding network providers, utilization of networks at their maximum capacity is considered a very vital factor behind business success. LTE is specifically designed for this particular purpose, and is furnished with several special characteristics, for example, it can be used in a variety of frequencies, has flexibility in terms of channel bandwidths, 
and can be used with both FDD and TDD. According to the aforementioned special characteristics, network providers are capable of utilizing the existing frequency effectively as we as providing more diversified services and give consumers greater satisfaction.

In order to fulfill the aforementioned objective and be able to shape such various characteristics, the technologies selected on the basis of 3GPP standards to be used for LTE air interface include Orthogonal Frequency Division Multiplexing (OFDM) and Multi-Input Multi-Output (MIMO), as well as utilization of higher-order modulation. This process can be carried out through enabling LTE to be capable of transmitting and receiving data at the maximum speed of $75 \mathrm{Mbit} / \mathrm{s}$ in the uplink and $300 \mathrm{Mbit} / \mathrm{s}$ in the downlink. Nevertheless, the actual utilization speed may differ from the theoretical value subjecting to several factors such as types of communication devices used, quality of channels, and amount of subscribers in that particular area [4].

\section{QOS PARAMETER}

The networks of 4G LTE hold a promise of performance that is comparable or even better than the broadband services provided by landline Wi-Fi access. However, even though the technical limits are high, due to the complexities associated with the cellular data networks and multimedia services as following:

\section{A. Voice over Internet Protocol (VoIP) on Mobile Phones}

VoIP is the technology for the delivery of voice communications over IP networks, such as the Internet. The channel used for voice communications must have low bandwidth. However, in order to make communications efficient, data should be divided into packets to allow low-latency packets to be transmitted and cause the smallest jitter. Here, the packet concerning voice communications is considered very important and the channel bandwidth, which is able to guarantee that packets will be transmitted within reasonable periods, must be determined. In addition, vital services, such as emergency calls and communications during emergencies, must be emphasized.

\section{B. Video Streaming}

On-demand video streaming applications which enable users to create real-time videos, such as YouTube and Hulu, are considered the significant factor encouraging network providers to provide quality networks. As for the sound quality and video streaming, the network must have a lot of bandwidth to supply users' needs. The streaming can be either of the person-to-person type which can share video in real time or of the content-to-person type. Each of the streaming types requires different quality of network service provision. For example, the latency must be more strongly emphasized in real-time video sharing, compared with content-to-person streaming. That is to say, real-time video sharing will require higher bandwidth for both uplink and downlink. Therefore, the quality of network service provision will depend on types of application streaming.

\section{Content Downloading}

The amount of bandwidth available to mobile phones, which is used in downloading movies, photos, and songs, differs from the real-time video streaming which can be operated in the batch mode. Here, the network has to adopt the best-effort scheduling approach in operation, and ensure that the lost packet will be retransmitted via a protocol such as TCP.

\section{Diversified Services and Applications}

There are a lot of game and social media applications on the mobile platform. Each of the applications requires different quality and means of service provision. For example, multiplayer games require rapidity and real-time response, whereas communications applications like Facebook only require adequate bandwidth for video or photo uploading.

Fig. 2 illustrates the four-layered QoS model based on the architecture proposed by ITU-T [5].

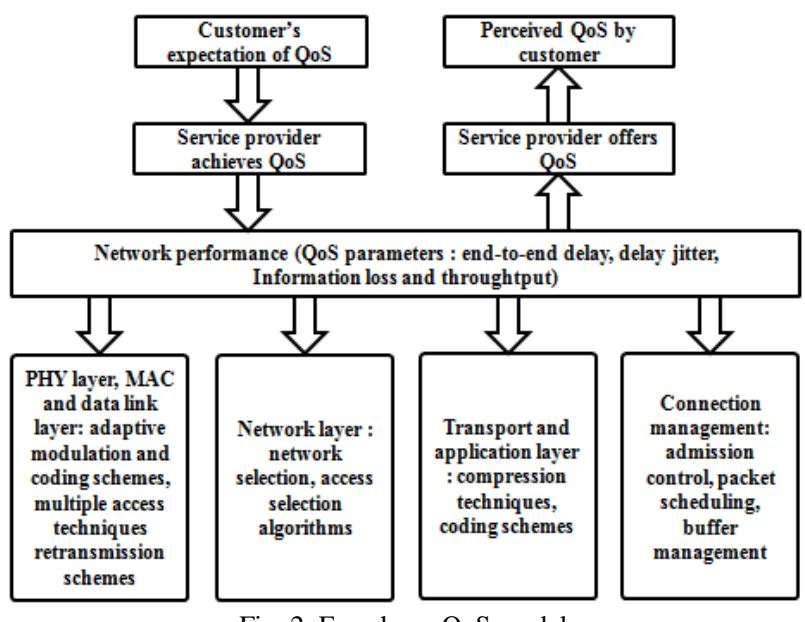

Fig. 2. Four-layer QoS model.

There is a large gap between engineering concepts and performance management in the QoS. Table I shows a comparison of the difference between nerwork performance aspect and QoS aspect [6].

\begin{tabular}{|l|l|}
\multicolumn{2}{|c|}{ TABLE I: NETWORK PERFORMANCE VS. QUALITY OF SERVICE [6] } \\
\hline \multicolumn{1}{|c|}{ Network Performance } & \multicolumn{1}{c|}{ Quality of Service } \\
\hline Provider oriented & User oriented \\
\hline Connection element attribute & Service attribute \\
\hline $\begin{array}{l}\text { Focus on planning, development, } \\
\text { operations and maintenance }\end{array}$ & Focus on user-observable effects \\
\hline $\begin{array}{l}\text { End-to-end or network connection } \\
\text { elements capabilities }\end{array}$ & Between at service access points \\
\hline
\end{tabular}

\section{RELATION BETWEEN QOS AND QOE}

Fundamentally, 4G LTE is designed to provide real-time, delay-sensitive multimedia to support a different type of Experience (QoE). QoE is a measurement of how well a system or an degradation in voice or video quality, whereas QoS focuses on standard quantitative performance from a network perspective.

Table II shows how subscriber QoE expectation varies by service type. It also describes the user's perception of quality on different services that have different performance attributes [7].

QoE is directly related to QoS. Therefore, one can map the objective QoS measurements (e.g.delay, packet loss and jitter) 
into the user's perception QoE, through an appropriate set of tools and processes. QoS is defined as the capability of the communication network to provide a service at an assured service level. QoE is basically depends on customer satisfaction in terms of usability, accessibility, and integrity of the service. QoE, however, is not limited to the technical performance of the network; there are also non-technical aspects, which influence the user perception and satisfaction. The Fig. 3 illustrates QoE is affected by the technical QoS and non-technical aspects of service [8].

TABLE II: COMPARISON OF QOE EXPECTATIONS AND PERFORMANCE REQUIREMENTS BY SERVICE TYPE [7]

\begin{tabular}{|c|c|c|}
\hline Services & QoE Expectation & $\begin{array}{l}\text { Performance } \\
\text { Attributes }\end{array}$ \\
\hline Internet & Low - best effort & $\begin{array}{l}\text { Variable bandwidth } \\
\text { consumption } \\
\text { Latency and loss } \\
\text { tolerant }\end{array}$ \\
\hline $\begin{array}{l}\text { Enterprise/Busines } \\
\text { s Services }\end{array}$ & High - critical data & $\begin{array}{l}\text { High bandwidth } \\
\text { consumption } \\
\text { Highly sensitive to } \\
\text { latency } \\
\text { High security }\end{array}$ \\
\hline Peer-To-Peer & Low - best effort & $\begin{array}{l}\text { Very- high bandwidth } \\
\text { consumption } \\
\text { Latency and loss } \\
\text { tolerant }\end{array}$ \\
\hline Voice & $\begin{array}{l}\text { High - Low latency and } \\
\text { jitter }\end{array}$ & $\begin{array}{l}\text { Low bandwidth - } \\
21-320 \text { Kbps per call } \\
\text { One-way latency < } \\
150 \mathrm{~ms} \\
\text { One-way jitter }<30 \mathrm{~ms}\end{array}$ \\
\hline Video & $\begin{array}{l}\text { High - low jitter and } \\
\text { extremely-low packet } \\
\text { loss }\end{array}$ & $\begin{array}{l}\text { Very-high bandwidth } \\
\text { consumption } \\
\text { Very sensitive to } \\
\text { packet loss }\end{array}$ \\
\hline $\begin{array}{l}\text { Gaming and } \\
\text { Interactive }\end{array}$ & $\begin{array}{l}\text { Services High }- \text { low } \\
\text { packet loss }\end{array}$ & $\begin{array}{l}\text { Variable bandwidth } \\
\text { consumption } \\
\text { One-way latency < } \\
150 \mathrm{~ms} \\
\text { One-way jitter }<30 \mathrm{~ms}\end{array}$ \\
\hline
\end{tabular}

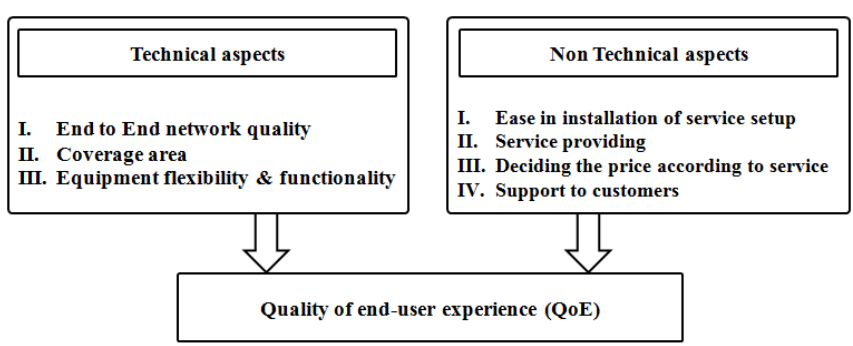

Fig. 3. Relation between QoS and QoE [8].

In 2014, IDA created an application for monitoring mobile phone service quality that gauges voice service to measure transmission delays and connection speeds of data service on various Internet technologies, i.e. 2G, 3G, 4G (LTE) and $\mathrm{WiFi}$, on specific service locations. It also asked subscribers to rate service satisfaction with their feedbacks reverted to IDA for in-depth analysis for the benefit of improving supervision and quality of the telecommunication industry. This testified to the priority given to tracking service quality, either Quality of Service (QoS) or Quality of Experience (QoE) which helped set efficient supervision policy.

Service quality in subscriber perspective, or QoE, is one of the main supervisory efficiency objectives. Raising QoE requires understanding of factors affecting subscriber satisfaction of related services, feedbacks of which could be tapped to further improve QoS in line with subscriber demand. QoS standards are essential tools for smooth operations of service net works and a guarantee of readiness to provide efficient data transmission and handle any emerging service disruption.

Determination of Internet QoS can often be made based on at least 4 factors, namely throughput, packet loss, delay and jitter, which greatly affect service quality from the perspective of Internet subscribers, as follows:

Throughput and actual bandwidth are telecommunication network capacity for accommodating traffic. Throughput can be tracked by actual volumes of time-based full file packets, measured in bit/s, that service networks can carry. Raising network bandwidth may help improve service quality, but focusing on adding bandwidth alone to rectify the problem may disproportionately incur additional operating cost as service efficiency can be developed and achieved by various other means, and it can be determined by the ratio of throughput or actual bandwidth and theoretical bandwidth (Table III).

TABLE III: THROUGHPUT STANDARD [9]

\begin{tabular}{|c|c|c|}
\hline & Category & $\begin{array}{c}\text { Throughput/Bandwidt } \\
\text { h }\end{array}$ \\
\hline $\begin{array}{c}\text { Throughput } \\
\text { Standard }\end{array}$ & Excellent & $\mathbf{1 0 0 \%}$ \\
\hline & Good & $\mathbf{7 5 \%}$ \\
\hline & Medium & $\mathbf{5 0 \%}$ \\
\hline & Poor & $<25 \%$ \\
\hline
\end{tabular}

Packet loss refers to failure to complete transmission of IP packets that is attributable to various factors, namely network capacity is overwhelmed by excessively heavy traffic demand for its existing bandwidth to handle. Packet loss measures packet failure as percentage of total actual packet traffic. If packet 1 osses exceed $15 \%$, they are regarded as below standards (Table IV).

\begin{tabular}{|c|c|c|}
\hline \multicolumn{2}{|c|}{ TABLE IV: PACKET LOSS STANDARD [10] } \\
\hline & Category & Packet Loss \\
\hline $\begin{array}{c}\text { Packet Loss } \\
\text { Standard }\end{array}$ & Excellent & $\mathbf{0 \%}$ \\
\hline & Good & $\mathbf{3 \%}$ \\
\hline & Medium & $\mathbf{1 5 \%}$ \\
\hline & Poor & $\mathbf{2 5 \%}$ \\
\hline
\end{tabular}

Delay or latency is the delay of point-to-point data transfer which may depend on transfer routes or distance involved, speeds of some service networks, e.g. the VoIP service for prompt, speedy data transfers which are highly important as any delay could disrupt flows of conversation to the point of making it impossible to proceed. Delays, attributable to various factors, can be measured by the ratio of packet length (bit) and link bandwidth (bit/s) . If the delay level is over 400 millisecond (ms), the network standard is regarded as below standard (Table V).

\begin{tabular}{|c|c|c|}
\multicolumn{2}{|c|}{ TABLE V: DELAY STANDARD [11] } \\
\hline Category & Delay \\
\hline Delay Standard & Good & $\mathbf{0 - 5} \mathbf{~ m s}$ \\
\hline & Medium & $\mathbf{1 5 0 - 4 0 0 ~} \mathbf{~ s}$ \\
\hline & Poor & $>\mathbf{4 0 0} \mathbf{~ m s}$ \\
\hline
\end{tabular}

Jitter refers to the state of instability experienced during data transfers, in other words varying degrees of delay seen in 
each packet. The fluctuating rate of delays in data transfers is called jitter which mostly occurs during packet switching, but which can be monitored by following the ratio of the sum of average delays in each packet (variable delay) and the volume of packet received, with the appropriate jitter level not exceeding $50 \mathrm{~ms}$ (Table VI).

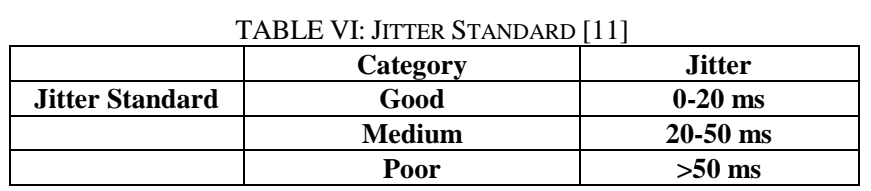

VoIP is voice communications run on the Internet or on other channels utilizing Internet protocol. The voice signals are divided into packets that are run on general communications networks, rather than on traditional telephone circuits.

\section{THE IMPACT OF QOS PARAMETER ON QOE}

The quality of telecommunication networks has material impact on QoE as far as the main Internet service is concerned. For example, VoIP, videoconferencing, video and audio streaming, client/server transactions, email and file transfers, all require varying network quality due to different sensitivity of subscriber experience to each service. [12], [13] (Table VII).

Real-time communication services, including VoIP and video conference, are highly sensitive to data transfer delays as slight such delays can disrupt services. Additionally, video conferencing requires high service quality relative to other service formats, while video and audio streaming, as well as client/server transactions demand median network quality, and email/file transfer services need relatively insignificant service quality although they are highly sensitive to packet losses.

TABLE VII: APPLICATION QOS METRICS SENSITIVITY [12]

\begin{tabular}{|c|c|c|c|c|}
\hline \multirow{2}{*}{ Application } & \multirow{2}{*}{ Throughput } & Delay & Jitter & $\begin{array}{c}\text { Packet } \\
\text { Loss }\end{array}$ \\
\cline { 3 - 5 } & Low & High & High & Medium \\
\hline VoIP & High & High & High & Medium \\
\hline $\begin{array}{c}\text { Video } \\
\text { Conferencing }\end{array}$ & High & Medium & Medium & Medium \\
\hline $\begin{array}{c}\text { Streaming } \\
\text { Video }\end{array}$ & Low & Medium & Medium & Medium \\
\hline $\begin{array}{c}\text { Streaming } \\
\text { Audio }\end{array}$ & Medium & Medium & Low & High \\
\hline $\begin{array}{c}\text { Client/Server } \\
\text { Transactions }\end{array}$ & Low & Low & Low & High \\
\hline Email & Medium & Low & Low & High \\
\hline File Transfer & & & \multicolumn{2}{|c|}{} \\
\hline
\end{tabular}

4G LTE operators can use the QoS metrices sensitivity for setting QoS standards for Internet connections in line with QoE standards which are essential for guaranteeing minimum quality that subscribers get and preventing service deterioration in the wake of service charge regulations enforced by relevant agencies. Setting QoS standards guarantees not only minimum Internet connection services that subscribers expect, disclosure of result of monitoring quality of each service provider not only can help subscribers choose service providers most suited to their requirements, but also encourage service providers to respond to market demand in developing and improving services.

\section{FUTURE WORK}

In the near future, the emergence of $5 \mathrm{G}$ mobile communication and networks technologies is revolutionizing the way people live and work. These technologies are moving towards a common set of objectives to offer the end users higher data rate, robustness, security, and QoS in a unified communication. $5 \mathrm{G}$ is the next step in the evolution of mobile communication and will be a key infrastructure of the mobile economy. International Telecommunication Union (ITU) has established the overall roadmap for the development of $5 \mathrm{G}$ mobile and defined the term it will apply to it as "IMT-2020". The roadmap of 5G shows in Fig. 4 [14].

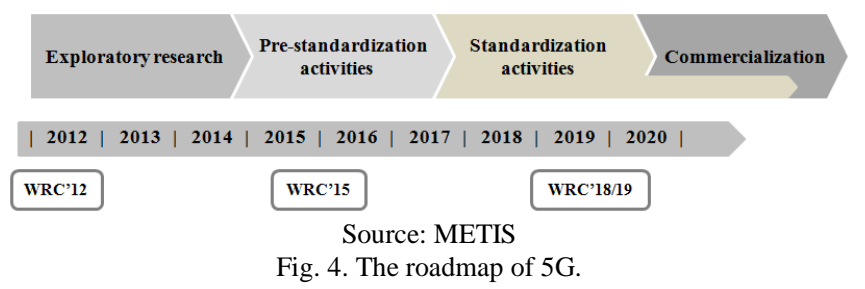

In order to provide end-to-end QoS support for application services over the converged networks in 5G, a common QoS framework will be proposed for the future research. As we discussed before in this paper, the QoS level is a major component of the perceived QoE. That is why QoS and QoE both will be studied from design to implementation in the $5 \mathrm{G}$ environment.

In hyperconnected 5G network, M2M scenario is considered as a major challenge for the $5 \mathrm{G}$ wireless cellular systems [15]. Hence, in addition to increased bit rate, 5G will be required to provide minimal latency to seamless integration of Internet of Things (IoT) nodes, and to support energy efficiency of the terminals and of the whole system as illustrated in Fig. 5 [16].

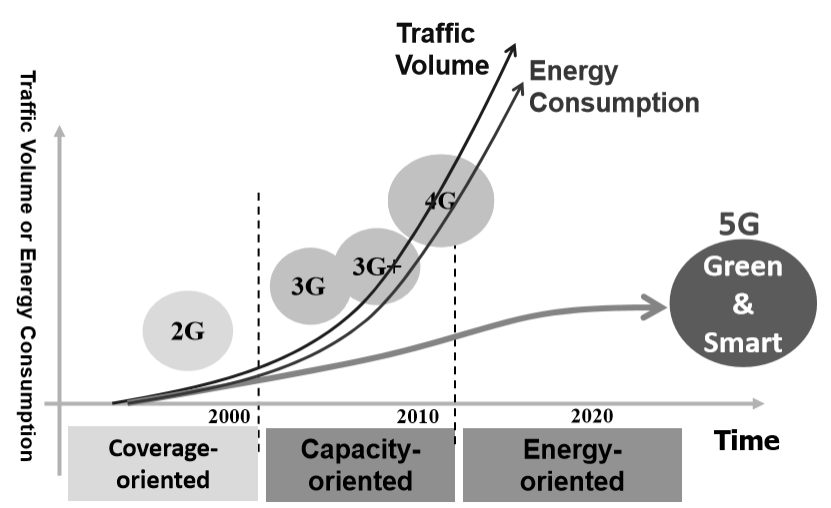

Fig. 5. Energy-oriented network in the next generation network [16].

Future mobile services will be grouped around video services and services based on the massive use of M2M devices in most industries and consumers' households [17]. According to forecasts, in 2018, the number of M2M connections in the mobile networks will exceed 1.5 billion, and mobile networks will have more than 2.6 billion M2M connections in 2020 [18]. Therefore, in 5G and M2M networks, the QoS level needs to meet the requirements 
resulting from future highly connected and automated devices and go beyond what can be achieved with any wireless communication technology today [19].

\section{CONCLUSION}

In the past, the provision of mobile services through $1 \mathrm{G}$ and $2 \mathrm{G}$ networks was focused on voice. Besides, the quality control in those services was less complicated than the quality control in providing $3 \mathrm{G}$ network services, especially in providing $4 \mathrm{G}$ LTE network services. This is because the means of service provision and mobile broadband services which are widespread motivate wireless network providers to transmit data through various applications, generating different characteristics of data traffic as a result. Successful telecommunications services can be achieved by managing QoE, attracting and retaining customers, improving user satisfaction and expanding the subscriber base. The challenge operators face today is how to monitor and improve QoS in real time and offer differentiated services. As for network operators, the customers' success will be characterized by their competency in offering these applications with the aim to bring customers great experiences. Consequently, network operators have to improve their means of service provision so as to gain the biggest return on investment (ROI) and execution. The provision of mobile phone networks has to be efficient and meets quality of services (QoS) in order to handle such challenges. Here, the LTE network, which is IP-based and has already become a widespread trend in mobile phone infrastructures, will help in acquiring more bandwidth and improving data latency.

\section{REFERENCES}

[1] M. Nohrborg, "LTE Overview," 3rd Generation Partnership Project.

[2] The Global mobile Supplier Association (GSA). Fast Facts. [Online]. Available: http://gsacom.com/paper/fast-facts-2/

[3] UMTS Forum, "Towards global mobile broadband: Standardising future of mobile broadband with LTE (long term evolution)," 2008.

[4] S. Parkvall, "Ericsson research," LTE - The Global Standard for Mobile Broadband, 2009.

[5] W. C. Hardy, QoS: Measurement and Evaluation of Telecommunications Quality of Service, Chichester, New York : Wiley, 2002.

[6] Tutorial Document on QoS and QoE, Internal Report, National Broadcasting and Telecommunications commission (NBTC), Dec. 2015

[7] (Dec. 2013). Quality of Service (QoS) and Policy Management in Mobile Data Networks, Validating Service Quality to Ensure Subscriber Quality of Experience (QoE). [Online]. Available: https://www.ixiacom.com/sites/default/files/resources/whitepaper/poli cy_m anagement.pdf

[8] C. V. Rao et al., Int. J. Comp. Tech. Appl., vol. 2, no. 3, pp. 639-645 May 2011

[9] Telkom Polytechnic, Throughput Standards.

[10] ETSI, Telecommunications and Internet Protocol Harmonization over Networks (TIPHON).

[11] International Telecommunications Union (ITU), ITU-T-G.114, General Characteristics of International Telephone Connections and International Telephone Circuits, 1998.
[12] Introduction to Quality of Service, Nortell Networks Corp., Ontario, Canada, 2003.

[13] A. Khalifeh et al., "QOS for multimedia applications with emphasis on video conferencing," Masters Project, Halmstad University, Feb. 2011.

[14] Roadmap and Workplan on Future Technologies (2020) from 3GPP, ITU and METIS.

[15] A. B. Marco et al., "The challenges of M2M massive access in wireless Cellular networks," Digital Communications and Networks, vol. 1, no. 1, Feb. 2015.

[16] Z.-S. Niu, "5G: A paradigm shift of cellular networks," Tsinghua National Lab for Information Science and Technology, Dec.4, 2014.

[17] V. Tikhvinskiy and G. Bochechka, "Prospects and QoS requirements in 5G networks," Journal of Telecommunications and Information Technology, Jan. 2015

[18] The Global M2M Market in 2013, Machina Research, London, Jan. 2013.

[19] R. Ratasuk, A. Prasad, Z. Li, A. Ghosh, and M. A. Uusitalo, "Recent advancements in M2M communications in $4 \mathrm{G}$ networks and evolution towards 5G," presented at 18th IEEE International Innovations in Services, Networks and Clouds (ICIN) Conference, Paris, Feb. 2015.

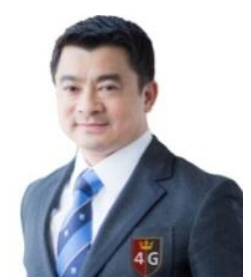

Settapong Malisuwan was born on March 24, 1966 in Bangkok, Thailand. $\mathrm{He}$ was awarded ful scholarship from Thai Government for Ph.D. in electrical engineering (telecommunications), specializing in mobile communication systems from Florida Atlantic University (State University System of Florida), Boca Raton in 2000. He received his MSc in electrical eng. in mobile communications system from George Washington University in 1996 and was awarded First Class Honors, Gold Medal Award and Outstanding Cadet Award by the university. $\mathrm{He}$ also achieved the MSc in electrical engineering in telecommunication engineering from Georgia Institute of Technology in 1992. Furthermore, he achieved military education from Special Warfare Center, Thailand, specializing in ranger and airborne courses in 1989 and 1988 respectively. $\mathrm{He}$ is currently the vice chairman and board commissioner of National Broadcasting and Telecommunications Regulator in Bangkok, Thailand. He was awarded the "Science towards the excellence in 2013" by the Senate Standing Committee on Science, Technology, Communications and Telecommunications. His research interests are in electromagnetics, efficient spectrum management and telecommunications policy and management.

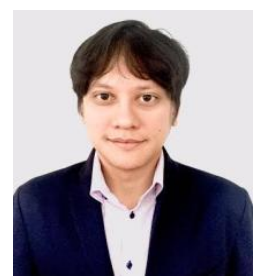

Dithdanai Milindavanij was born in Bangkok, Thailand on February 15, 1981. He received his master of political science in political management from Ramkhamhaeng University in 2006. He has been working in National Broadcasting and Telecommunications, Bangkok, Thailand Office since 2011 and has been working as an assistant to vice chairman in National Broadcasting and Telecommunications since May 2015. His research interests are in technology management and spectrum management.

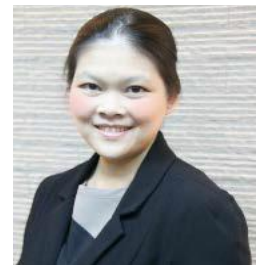

Wassana Kaewphanuekrungsi was born on February 1, 1979, Bangkok, Thailand. She received her B.S. in statistics with a major in business information technology from Chulalongkorn University, Thailand, 2000, and the M.S. in technology management from Thammasat University, Thailand, 2006. She joined National Broadcasting and Telecommunication Commission (NBTC) as assistance to vice chairman of NBTC in December 2011, which is responsible in the field of telecommunications research. Previously in 2010, she worked for as senior system analyst at Bangkok Insurance (Public) Co., Ltd. Her research interests are in information technology, telecommunication technology, technology management and spectrum management. 